\section{GW23-e0830 A PRELIMINARY STUDY ON TEST EFFICIENCY OF ARTERIAL FUNCTION PARAMETERS IN NORMAL YOUTHS BY HIGH FREQUENCY ULTRASOUND COMBINED WITH EXERCISE STRESS TESTING}

doi:10.1136/heartjnl-2012-302920ad.31

Zhao-jun Li, Chun-song Kang, Yan-xia Zhang, Ji-ping Xue, Kai-ling Shi, Chunsong Kang. Department of Ultrasound, Shanxi Academy of Medical Sciences \& Shanxi DAYI Hospital

Objectives To investigate the difference of carotid elasticity function by two-dimensional speckle tracking imaging (X-strain) combined with ultrasound RF signal vascular stiffness (Quantitative Arterial Stiffness, OAS) technique, before and after exercise stress test, analysis the difference between the left and right carotid artery function, and find function index that can sensitively reflect changes.

Methods 49 healthy young people were enrolled in this study, exercise stress test by using the improved Bruce scheme. Before and after exercise stress test, arterial function parameters were measured using OAS technology including arterial compliance (CC), arterial distensibility (DC), stiffness parameter $\alpha, \beta$ and pulse wave velocity (PWV); while the parameters reflecting arterial strain including endovascular circumferential strain (EN-CS), endovascular circumferential strain rate (EN-CSR), adventitial circumferential strain (EP-CS), adventitial circumferential strain rate (EP-CSR) were collected using the technique of X-strain. The parameters were compared before and after exercise, analysis the difference between the left and right carotid artery function, ROC curves were drawn by the basal arterial parameters before the exercise, comprising test efficiency of these parameters.

\section{Results}

1. Arterial function parameters CC, DC were lower (1.04 \pm 0.43 vs $1.44 \pm 0.45 \mathrm{~mm}^{2} / \mathrm{kPa}, 0.03 \pm 0.01$ vs $\left.0.04 \pm 0.021 / \mathrm{kPa}, \mathrm{p}<0.05\right)$, while $\alpha, \beta$ and PWV were higher $(1.19 \pm 0.17$ vs $0.63 \pm 0.09,2.51$ \pm 0.36 vs $1.28 \pm 0.18,5.84 \pm 1.16$ vs $5.04 \pm 0.79 \mathrm{~m} / \mathrm{s}, \mathrm{p}<0.05)$ than that before the exercise; EN-CS, EN-CSR, EP-CS, EP-CSR were higher $\left(9.10 \pm 4.23\right.$ vs $7.83 \pm 2.45 \%, 1.22 \pm 0.56$ vs $0.83 \pm 0.28 \mathrm{~S}^{-1}$, $7.94 \pm 3.63$ vs $6.89 \pm 2.47 \%, 1.02 \pm 0.41$ vs $\left.0.71 \pm 0.25 \mathrm{~S}^{-1}, \mathrm{p}<0.05\right)$ than that before the exercise.

2. Either before or after exercise stress test, CC, DC, $\alpha, \beta$, and PWV had no difference between the left and right carotid artery $(p>0.05)$, EN-CS, EN-CSR, EP-CS, EP-CSR were the same $(p>0.05)$.

3. The ROC curve analysis showed that, the area under the curve of CC $>\alpha>\beta>$ PWV $>$ DC, respectively $0.782,0.774,0.763,0.702$, $0.691(p<0.05), Z$ test showed no significant difference between the parameters $(p>0.05)$; area under the curve of EP-CSR $>E N$ CSR $>$ EP-CS $>$ EN-CS, respectively.0.742 $\quad(p<0.05), 0.724$ $(p<0.05), 0.585(p>0.05), 0.565(p>0.05), Z$ test showed that ENCSR and EP-CSR have no significant difference ( $p>0.05)$.

4. The test efficiency of CC, DC, $\alpha, \beta$, PWV and EN-CSR, EP-CSR reflecting changes in vascular function has no significant difference $(p>0.05)$.

Conclusions The changes of arterial function before and after exercise are physiological. Arterial function between the left and right carotid artery have no difference. Ultrasonography arterial function parameters CC, DC, $\alpha, \beta$, PWV, EN-CSR and EP-CSR have higher test efficiency than others. 